November 29, 1989

CMU-HEP 89-23

MPI-PAE/PTh $75 / 89$

\title{
Majorons and Supernova Cooling
}

\author{
Kiwoon Choi ${ }^{1)}$ and A. Santamaria* 2) \\ 1) Department of Physics, Carnegie Mellon University \\ Pittsburgh, PA 15213, USA \\ 2) Max-Planck-Institut für Physik und Astrophysik \\ Föhringer Ring 6, D-8000 München 40, Germany
}

\begin{abstract}
We consider the role of majoron emission in supernova cooling and its implications for the neutrino mass and lifetime in generic singlet majoron models. It is found that for $\nu_{\tau}$ with mass $m$, if the lifetime for the decay $\nu_{\tau} \rightarrow$ majoron $+\nu_{e, \mu}$ is shorter than $10^{-7}(\mathrm{~m} / \mathrm{MeV}) \mathrm{sec}$, then majorons are so strongly trapped by the inverse process that the resulting majoron luminosity is small enough to not destabilize the observed $\nu_{e}$-pulse from SN1987A. For $\nu_{\tau}$ with a longer lifetime, the majoron luminosity can be large enough to destroy or significantly shorten the duration of the neutrino pulse. We then find the range of parameters, e.g. the $\nu_{\tau}$-mass $m$ and the $B-L$ breaking scale $v$, that is excluded by giving such a large majoron luminosity. Our results imply that, for $v$ between $1 \mathrm{GeV}$ and $1 \mathrm{TeV}$, a wide range of $m$ allowed by terrestrial experiments can be excluded in view of the observed $\nu_{e}$-pulse from SN1987A.
\end{abstract}

*Permanent address: Departament de Física Teòrica, Universitat de València and IFIC, Universitat de València-CSIC, Spain. 


\section{Introduction}

The supernova SN1987 $\mathrm{A}^{[1]}$ in the Large Magelanic Cloud has provided a lot of information on the properties of neutrinos, e.g. masses, number of generations ${ }^{[2]}$, mag-

netic moments ${ }^{[3]}$, and exotic interactions ${ }^{[4-8]}$. One clear observation associated with SN1987A is the thermal neutrino pulse (more precisely $\nu_{e}$-pulse) which is considered to carry off most of the gravitational binding energy of the resulting neutron star. As a result, any exotic interaction of neutrinos must be tuned to not destabilize this neutrino pulse.

In majoron models ${ }^{[9-13]}$, where neutrinos have nonzero Majorana masses due to the spontaneous $B-L$ violation, there exist exotic interactions of neutrinos with the Higgs fields, in particular with the massless majoron component $\phi$, that trigger the spontaneous $B-L$ violation. The implications of these additional interactions for the dynamics of supernova neutrinos have already been considered by many authors. The energy release by majoron emission may significantly shorten the duration of the neutrino pulse from supernovae $e^{[6,7]}$. Too much neutrino-majoron scattering inside the supernova core would delay neutrino emission ${ }^{[8]}$. Also the scattering between supernova neutrinos and cosmic background majorons will lead to an energy loss for the neutrinos and thus effectively stops them being detected ${ }^{[4]}$. Among these implications, in this paper, we will concentrate on the role of majoron emission in the cooling of the hot nascent neutron star associated with SN1987A.

In Ref. 6, the emission of majorons from supernovae through the process $\nu \nu \rightarrow \phi \phi$ has been considered with the assumption that only the coupling of the form $h \phi \bar{\nu} i \gamma_{5} \nu$ is responsible for the majoron production process, viz all other majoron couplings were assumed to be weak enough. Then it was found that for a majoron-neutrino Yukawa 
coupling $h$ in the range $\mathrm{O}\left(10^{-5}\right) \leq h \leq \mathrm{O}\left(10^{-4}\right)$, the majoron luminosity dominates over the neutrino luminosity, which seems to be inconsistent with the observed neutrino pulse from SN1987A. However as we will see in Appendix C, the assumption made in Ref. 6 is valid only for a special range of parameters as is the corresponding conclusion about $h$. The matter-induced decay $\nu \rightarrow \nu \phi$ was discussed in Ref. 7 as another process that produces majorons inside the supernova core, however only for neutrinos with $m \ll G_{F} Y_{n}\left(Y_{n}=\right.$ number density of nucleons inside the supernova).

Furthermore none of the above mentioned papers provided a complete analysis of the possible trapping of majorons. Although the authors of Ref. 6 took into account the process $\phi \nu \rightarrow \phi \nu$ and the matter-induced majoron absorption $\phi \nu \rightarrow \nu$ was considerd in Ref. 7, there always exists a possibility that majorons are strongly trapped by other processes. Then in order to find the forbidden region of the parameter space where the majoron luminosity is large enough to destablize the neutrino pluse from SN1987A, one should take into account all the processes that may trigger the trapping of majorons. Note that if majorons are strongly trapped by anyone of the processes under consideration, the resulting majoron luminosity will be small and will not affect the neutrino pulse regardless of the strength of the other processes. It is therefore tempting to analyze supernova cooling via majoron emission in a fully general context, particularly to analyze the effects of all the possible majoron interactions on the trapping of majorons inside the supernova core. The purpose of this paper is to provide such an analysis.

For majoron models in which the massless majoron belongs mainly to an electroweak non-singlet Higgs field ${ }^{[12,13]}$, e.g. the triplet model of Gelmini and Roncadelli, majorons are strongly trapped by the weak neutral current interactions with background nucleons. Furthermore in such models, the global $U(1)_{B-L}$ symmetry is prob- 
ably restored inside the supernova core since the astrophysical bound on the majoronelectron coupling ${ }^{[14,15]}$ (see eq.(2)) gives a severe constraint on the $B-L$ breaking scale $v$, viz $v \leq \mathrm{O}(10) \mathrm{KeV} \ll T$ ( $T=$ core temperature of the supernova). Thus, throughout this paper, we consider only models in which the majoron belongs mainly to an electroweak singlet Higgs field ${ }^{[9-11]}$. Note that the magnitude of $v$ in generic singlet majoron models is not highly constrained and can be arbitrarily large compared to the core temperature of the supernova.

Majoron emission from hot stars must be constrained in view of the long burning time scale. Among the various majoron couplings that induce majoron production, the role of the majoron electron coupling of the form

$$
\frac{1}{2} g_{\phi e e} m_{e}^{-1} \partial_{\mu} \phi \bar{e} \gamma^{\mu} \gamma_{5} e
$$

has been studied well (together with the axion coupling of the same form) and leads to the upper bound ${ }^{[14,15]}$

$$
\left|g_{\phi \mathrm{ee}}\right| \leq 1.4 \times 10^{-13}
$$

Although this bound strongly constrains the scale $v$ in gauge non-singlet majoron models by $v \leq \mathrm{O}(10) \mathrm{KeV}$, it says little about singlet majoron models since it is easily satisfied for the natural values of parameters in the theory.

One unique property of the majoron is that it can have relatively strong interactions with the neutrino while keeping the couplings to ordinary matter, e.g. electrons or nucleons, weak enough to satisfy the astrophysical bound of eq.(2). Furthermore, the coupling of the majoron to neutrinos is simply determined by the neutrino mass $m$ and the $B-L$ breaking scale $v$, and thus any information about it can be translated into a constraint on either $m$ or $v$. It would therefore be very interesting to have an astrophysical constraint on the majoron interactions with neutrinos. Then supernovae 
provide a unique way to get astrophysical information about the majoron-neutrino coupling, because of the presence of the thermal neutrino sphere ${ }^{[16]}$ that contains a high density of neutrinos for a relatively long time scale of $5 \sim 10 \mathrm{sec}$.

Majoron emission from SN1987 $\mathrm{A}^{[17]}$ can also be constrained by the observed neutrino pulse. It is generally believed that the remnant of SN1987A was a neutron star whose gravitational binding energy has an upper limit of $6 \times 10^{53} \mathrm{erg}$. The relatively long time scale $(t=5 \sim 10 \mathrm{sec})$ of the neutrino flux with total energy $E_{\text {tot }}^{\nu}=(1 \sim 4) \times 10^{53} \mathrm{erg}$ then severely constrains majoron emission. If the majoron luminosity $L_{\phi}$ was greater than $10^{53} \mathrm{erg} / \mathrm{sec}$, the neutrino pulse would be affected significantly, perhaps enough to be inconsistent with the observed neutrinos. In this paper, as a conservative bound, we will take $3 \times 10^{53} \mathrm{erg} / \mathrm{sec}$ as the maximum allowed majoron luminosity, viz

$$
L_{\phi} \leq 3 \times 10^{53} \mathrm{erg} / \mathrm{sec}
$$

and try to find the parameter region excluded by eq.(3). This simple approach has turned out to be a good approximation to a more careful analysis including the effects of majoron emission on the detailed models for supernova dynamics ${ }^{[18]}$.

If majorons interact with background particles so weakly that the majoron mean free path $l_{\phi}$ is greater than the radius $r_{0}$ of the inner core, then majorons will freely stream out from the supernova. In this case, the majoron luminosity $L_{\phi}$ is proportional to the majoron creation rate times the volume of the hot core region (volume emission). The total creation rate for freely escaping majorons is a simple sum of the partial creation rates for each of the relevant processes. Then in considering the parameter range that gives $L_{\phi} \geq 3 \times 10^{53} \mathrm{erg} / \mathrm{sec}$, one can consider only a particular set of interesting processes without worrying about the role of the other processes. The corresponding range of parameters must be ruled out regardless of the ignored processes. For relatively strong majoron couplings that give $l_{\phi} \ll r_{0}$, majorons are trapped inside 
the supernova core and form a thermal sphere of radius $R_{\phi}$ at which the majoron optical depth is of order unity. Then $L_{\phi}$ can be approximated by blackbody emission from this majoron sphere (blackbody surface emission). A crucial point in the case of surface emission is that one must take into account all of the processes that are potentially relevant to majoron trapping in order to find the parameter region excluded by giving $L_{\phi} \geq 3 \times 10^{53} \mathrm{erg} / \mathrm{sec}$.

The organization and summary of this paper are as follows. First of all, in Sec. 2, we present an effective lagrangian describing majoron interactions with neutrinos, electrons and nucleons that are copious inside supernovae. Since we are interested only in singlet majoron models, we adopt the singlet majoron model of Chikashige, Mohapatra, and Peccei (CMP) ${ }^{[9]}$ as a guideline in deriving our effective lagrangian. However the final form will be general enough so that it can be applied to a wide class of majoron models. In Sec. 3, majoron luminosities for both volume emission and blackbody surface emission will be computed in terms of our effective lagrangian. For volume emission, as processes producing majorons inside the supernova core, we consider only those associated with the majoron couplings to neutrinos, viz $\nu \nu \rightarrow \phi \phi$, $\nu \rightarrow \nu^{\prime} \phi$ and $\nu \rightarrow \nu \phi$. Here $\nu$ denotes the heaviest neutrino (presumed as $\nu_{\tau}$ in what follows) while $\nu^{\prime}$ is a lighter neutrino (either $\nu_{\mu}$ or $\nu_{e}$ ). The last process is due to the effect of background matter. As processes that contribute to majoron trapping, we consider all the potentially relevant ones including those associated with majoronnucleon couplings, e.g. $\phi \nu \rightarrow \phi \nu, \phi \phi \rightarrow \nu \nu, \phi \phi \rightarrow \phi \phi, \phi n \rightarrow \phi n, \phi \nu^{\prime} \rightarrow \nu$ and $\phi \nu \rightarrow \nu$. Again here the last process is the result of matter effects. We also argue that for the class of singlet majoron models under consideration, the effects of the processes $\phi n n \rightarrow n n$ and $\phi n \rightarrow \phi \phi n$ to majoron trapping is negligible.

The present experimental upper bound on the mass of the $\nu_{\tau}$ is $35 \mathrm{Mev}$ and the 
constraint on its lifetime, coming from cosmological arguments, is very mild ${ }^{[19]}$. Our results provide information on the phenomenologically allowed range of the mass $m$ and lifetime $\tau_{\nu}$ of $\nu_{r}$ in majoron models that is extremely hard to obtain in terrestrial experiments. In particular if $\tau_{\nu} \geq 10^{-6}(\mathrm{~m} / \mathrm{MeV})$ sec and $v$ is $\mathrm{O}(1) \mathrm{GeV}$ to $\mathrm{O}(1) \mathrm{TeV}$, a wide range of $m$ gives a majoron luminosity greater than $3 \times 10^{53} \mathrm{erg} / \mathrm{sec}$ and thus must be ruled out. This excluded range of $m$ depends on $v$ and is depicted in Fig. 5 .

Recently some singlet majoron models with $v$ at the Fermi scale have been suggested for the purpose of realizing neutrino masses not far below the present experimental limit ${ }^{[20]}$ or of avoiding potential hierarchy problems ${ }^{[1]}$. Furthermore, more recently Carlson and Hall ${ }^{[21]}$ considered the possibility of having neutrinos as dark matter ${ }^{[2]}$ in the context of the CMP model with $v \simeq \mathrm{O}(1) \mathrm{GeV}$. Our analysis can be relevant to these models.

After our conclusion and summary in Sec. 4, Appendix A provides explicit formulae for various scattering cross-sections. In Appendix B, we discuss the effects of background matter on neutrino propagation inside supernovae, and in Appendix $\mathrm{C}$, we consider the region of the parameter space where the result of Ref. 6 is valid.

\section{Effective Lagrangian for Majoron Interactions}

As was explained, we are interested in majoron dynamics inside the supernova core. Then the characteristic energy of majorons is $\mathrm{O}(10 \sim 100) \mathrm{MeV}$ and the relevant particles are neutrinos, electrons and nucleons. In this section we consider the effective lagrangian that describes majoron interactions with those particles that are copious inside supernovae in the context of the CMP model. For the later discussion of the matter-induced neutrino decay, we will include terms which are the results of the coher- 
ent neutrino scattering off background nucleons. Although obtained within the CMP model, the final form of our effective lagrangian is valid for other majoron models.

The CMP model contains a gauge singlet complex scalar field $\sigma$ with $B-L=2$ and also three flavors of singlet right-handed neutrinos $N_{R}$ with $B-L=-1$ (in a threefamily model) in addition to those of the minimal standard model. At the lagrangian level, $U(1)_{B-L}$ is an exact symmetry and the part of the lagrangian that involves these additional fields is

$$
\partial_{\mu} \sigma \partial^{\mu} \sigma^{*}-\left(\frac{1}{\sqrt{2} v} \sigma^{*} \bar{N}_{R} M_{1}\left(N_{R}\right)^{c}+\frac{2}{\sqrt{2} v_{2}} H_{i} \bar{N}_{R} M_{2} L^{i}+h . c .\right)-V(\sigma, H)
$$

where $L^{i}$ ( $i=1,2=$ weak isospin index) denotes the leptonic doublet and $H_{i}$ is the standard Higgs doublet. Here $\left(N_{R}\right)^{c} \equiv C{\overline{N_{R}}}^{T}$ is the charge conjugated spinor. We are using four component notation for all spinors, and their flavor indices are suppressed. In what follows, we shall assume that both $M_{1}$ and $M_{2}$ are real matrices and thus ignore $C P$-violating effects.

The Higgs potential $V$ takes the form

$$
\lambda_{1}\left(H^{\dagger} H-\frac{v_{2}^{2}}{2}\right)^{2}+\lambda_{2}\left(H^{\dagger} H-\frac{v_{2}^{2}}{2}\right)\left(\sigma \sigma^{*}-\frac{v^{2}}{2}\right)+\lambda_{3}\left(\sigma \sigma^{*}-\frac{v^{2}}{2}\right)^{2},
$$

with $\lambda_{1}$ and $\lambda_{3}$ positive. Then for $4 \lambda_{1} \lambda_{3}>\lambda_{2}^{2}$, we have $\left\langle H_{i}\right\rangle=v_{2} \delta_{i}^{1} / \sqrt{2}$ and $\langle\sigma\rangle=v / \sqrt{2}$. The massless majoron field $\phi$ belongs to $\sigma$ and can be identified as

$$
\sigma=\frac{1}{\sqrt{2}}(v+\rho) \exp (i \phi / v)
$$

where $\rho$ denotes a massive scalar partner of the majoron with mass-squared $m_{\rho}^{2}=2 \lambda_{3} v^{2}$.

Even though the $B-L$ breaking scale $v$ in the CMP model was usually considered as higher than the electroweak scale $v_{2}$, it has recently been argued ${ }^{[20,21]}$ that the model with $v$ less than or close to $v_{2}$ can have various kinds of interesting phenomenology. 
Furthermore, for other types of singlet majoron models ${ }^{[11]}$, there is no theoretical or phenomenological argument that constrains $v$ to be above $v_{2}$. We therefore keep our analysis general so that it can be applied to models of relatively low $B-L$ breaking scale. In particular we will include $\rho$ explicitly in our later discussions of the majoron effective lagrangian. Note that $m_{\rho}$ can be even significantly less than $v$ for models with small quartic couplings.

Let us first consider the interaction lagrangian involving the majoron $\phi, \rho$ and neutrinos only. Then we have

$$
\begin{gathered}
\frac{1}{2}\left(\partial_{\mu} \rho\right)^{2}+\frac{1}{2}(1+\rho / v)^{2}\left(\partial_{\mu} \phi\right)^{2}-\frac{\lambda_{3}}{4}\left(4 v^{2} \rho^{2}+4 v \rho^{3}+\rho^{4}\right) \\
-\left(\bar{N}_{R} M_{2} N_{L}+\frac{1}{2}(1+\rho / v) \bar{N}_{R} M_{1}\left(N_{R}\right)^{c}+h . c .\right) \\
+\frac{1}{2 v} \partial_{\mu} \phi\left(\bar{N}_{R} \gamma^{\mu} N_{R}+\bar{N}_{L} \gamma^{\mu} N_{L}\right)-\frac{1}{\sqrt{2}} G_{F} Y_{n} u_{\mu} \bar{N}_{L} \gamma^{\mu} N_{L},
\end{gathered}
$$

where $N_{L}$ are the neutrinos in the weak lepton doublets and $Y_{n}$ is the number density of background nucleons with collective four-velocity $u_{\mu}$. The last term in eq.(7) denotes the effect of coherent neutrino forward scattering ${ }^{[23]}$ off background nucleons via the standard weak interactions. It can be obtained by replacing the nucleon current $J^{\mu}(n)$ by $Y_{n} u^{\mu}$ in the effective four-fermion interaction term $G_{F} J^{\mu}(n) \bar{N}_{L} \gamma_{\mu} N_{L} / \sqrt{2}$ which is induced by the standard weak interactions. Note that, for $\nu_{\mu}$ and $\nu_{\tau}$, the coherent neutrino scattering arises mainly from the neutral current interactions with nucleons. We have replaced the Higgs doublet by its VEV and performed the majoron dependent transformation of the neutrino fields

$$
N_{R} \rightarrow N_{R} \exp (-i \phi / 2 v), N_{L} \rightarrow N_{L} \exp (-i \phi / 2 v)
$$

in arriving at the above form of the effective lagrangian.

Let us now adopt the usual assumption for the seesaw mechanism

$$
M_{1} \gg M_{2} \text {. }
$$


Then the diagonalization of the whole mass matrix leads to three heavy Majorana neutrinos, denoted by $\nu_{H}$ in what follows, and three light ones. The heavy neutrinos $\nu_{H}$ are mostly gauge singlets and have a mass $\sim M_{1}$, and the light neutrinos are mostly doublets and their mass is given by $\sim-M_{2}^{T} M_{1}^{-1} M_{2}$. The heavy neutrinos $\nu_{H}$ may also have interesting effects on supernova dynamics, however we will not consider this aspect here and simply omit $\nu_{H}$ with the assumption that their masses are large compared to the supernova temperature.

To proceed, let $\nu$ and $\nu^{\prime}$ denote the heaviest component (presumably $\nu_{\tau}$ ) and the lighter one ( either $\nu_{\mu}$ or $\nu_{e}$ ) with masses $m$ and $m^{\prime}$ respectively among the light neutrinos that are mostly weak doublet. Then, omitting $\nu_{H}$, the majoron interactions with $\rho, \nu$ and $\nu^{\prime}$ which are relevant to our discussion of majoron emission from supernovae and the operators composed of neutrino fields which represent the background matter effects can be written as

$$
\begin{gathered}
\frac{\rho}{v}\left(\partial_{\mu} \phi\right)^{2}+\frac{1}{2} b_{\mu}\left(\bar{\nu} \gamma^{\mu} \gamma_{5} \nu+\overline{\nu^{\prime}} \gamma^{\mu} \gamma_{5} \nu^{\prime}-X \bar{\nu} \gamma^{\mu} \gamma_{5} \nu^{\prime}\right) \\
-\frac{1}{2 v} \rho\left(m \bar{\nu} \nu+m^{\prime} \bar{\nu}^{\prime} \nu^{\prime}-X\left(m+m^{\prime}\right) \bar{\nu} \nu^{\prime}\right) \\
-\frac{1}{4 v} \partial_{\mu} \phi\left(\bar{\nu} \gamma^{\mu} \gamma_{5} \nu+\bar{\nu}^{\prime} \gamma^{\mu} \gamma_{5} \nu^{\prime}-2 X \bar{\nu} \gamma^{\mu} \gamma_{5} \nu^{\prime}\right)
\end{gathered}
$$

where the first term denotes the majoron coupling to $\rho$ with $m_{\rho}^{2}=2 \lambda_{3} v^{2}$ and the terms with the coefficient $b_{\mu}=G_{F} Y_{n} u_{\mu} / \sqrt{2}$ are due to background matter effects. In deriving this expression we kept only the dominant term (in an expansion in $M_{2} / M_{1}$ ) for each type of coupling. The value of $X$ depends on the detailed form of the matrices $M_{1}$ and $M_{2}$ but for $M_{2} \ll M_{1}$ we will have

$$
X \ll 1
$$

The value of $X$ is particularly interesting in connection with the $\nu$-lifetime in the vacuum. In fact, with a plausible ansatz for the structure of $M_{2} M_{2}^{T}$ in the CMP model, 
Glashow ${ }^{[20]}$ estimated that, for the coupling $\partial_{\mu} \phi \overline{\nu_{\tau}} \gamma^{\mu} \gamma_{5} \nu_{\mu}$ with the mixing parameter $\alpha \leq 0.1$ (see Ref. 20 for the definition of $\alpha$ ),

$$
X \leq 8.1 \times 10^{-5}\left(\frac{m}{M e V}\right)\left(\frac{m^{\prime}}{K e V}\right)
$$

The easiest way to find the relevant couplings involving matter fermions $\psi=$ $(e, p, n)$ would be to start with the $C P$ and $B-L$ invariant effective vertices of the following form, induced by weak gauge boson or Higgs boson exchange:

$$
i G_{F}\left(\sigma \partial_{\mu} \sigma^{*}-\sigma^{*} \partial_{\mu} \sigma\right) \bar{\psi} \gamma^{\mu}\left(V+A \gamma_{5}\right) \psi+G_{F}\left(\sigma \sigma^{*}-\frac{v^{2}}{2}\right) \bar{\psi} S M_{\psi} \psi
$$

Here $M_{\psi}$ is the mass matrix of $\psi=(e, p, n), G_{F}$ is the Fermi constant, and $V, A$ and $S$ denote dimensionless effective coupling constants that are calculable in terms of the fundamental parameters of the theory. Note that $B$ and $L$ symmetries imply that the matter fermions $\psi$ can couple to $\sigma$ only through nonrenormalizable terms. Then again with the parameterization of eq.(6), we obtain

$$
G_{F} v \partial_{\mu} \phi(1+\rho / v)^{2} \bar{\psi} \gamma^{\mu}\left(V+A \gamma_{5}\right) \psi+\frac{1}{2} G_{F}\left(2 v \rho+\rho^{2}\right) \bar{\psi} M_{\psi} S \psi
$$

In the CMP model, the coupling of the majoron to the bilinear current of $\psi$ is induced by the diagram of Fig. 1 where the four-fermion vertex is due to the weak gauge boson exchange. The resulting values of the effective coupling constants $V$ and $A$ can be easily read off from Ref. 9. The coupling of $\rho$ to the scalar density of $\psi$ is the result of the mass mixing between $\rho$ and the physical Higgs in $H_{i}$, which is induced by the term in the Higgs potential of eq.(5) having the coefficient $\lambda_{2}$. In the case of $v \ll v_{2}$, the diagram of Fig. 2 representing the effective coupling of $\rho$ to electrons or quarks eventually gives rise to an effective coupling constant $S=\operatorname{diag}\left(S_{e}, S_{p}, S_{n}\right)$, with

$$
S_{e}=\frac{\lambda_{2}}{m_{H}^{2} G_{F}}, S_{p}=S_{n}=\frac{\lambda_{2}}{2 m_{N} m_{H}^{2} G_{F}}\left(\frac{2 m_{s}}{m_{u}+m_{d}} \sigma_{\pi N}+2 m_{N}-m_{\Sigma}-m_{\Xi}\right),
$$


where $m_{H}$ denotes the mass of the Higgs doublet, $m_{u}, m_{d}$ and $m_{s}$ are the current quark masses, $m_{N}, m_{\Sigma}$ and $m_{\Xi}$ are the baryon masses and $\sigma_{\pi N} \simeq 50 \mathrm{MeV}$ is the pion nucleon $\sigma$-term. In the opposite limit of $v \gg v_{2}$ which is usually considered to be the case for the CMP model, the corresponding values of $S$ are suppressed by the factor $\left(v_{2} / v\right)^{2}$ compared to those in eq.(15).

If the $B-L$ breaking scale is above the electroweak scale, viz $v>v_{2}$, then $\rho$ may be heavy enough so that, inside the supernova core, the effects of the processes involving real or virtual $\rho$ 's are strongly suppressed. In this case, one can simplify the analysis by putting $\rho=0$ in our effective lagrangian. One should note that this simple way to realize the decoupling of a heavy $\rho$ is allowed only with the exponential parameterization of $\sigma$ in eq.(6) (See Appendix C).

In the next section, we will consider majoron emission from supernovae with the effective lagrangian considered above. Even though it is derived within the CMP model, one can always obtain a similar form of the effective lagrangian for other majoron models, and thus any result based on it can be applied to a wide class of models. In this respect, we will use the estimate of the effective coupling constants, e.g. $A, S, X$ etc., obtained within the CMP model as a guideline and not as a constraint.

\section{Energy Release by Majoron Emission}

In this section, we consider supernova cooling via majoron emission in the context of the effective lagrangian derived in the previous section. We first consider the majoron mean free path inside the supernova core and then the majoron luminosity in the cases of volume emission and blackbody surface emission will be computed together with the resulting constraints. 
For simplicity, we also make the following assumptions about the parameters in our effective lagrangian: (1) $m \gg m^{\prime}$; (2) $A \simeq V$; (3) $A_{e} \simeq A_{p} \simeq A_{n}$ and $S_{e} \simeq S_{p} \simeq S_{n}$. In view of the large hierarchy in the mass spectrum of charged leptons, the first assumption is quite plausible and the second and third assumptions hold true for a wide class of models including the CMP model.

At the time period of thermal neutrino emission ${ }^{[16]}$ that concerns us here, it is believed that the electron number density (equal to the proton number density) is about $1 / 3 \sim 1 / 4$ of the neutron number density. Furthermore for the inner core temperature $T_{0} \simeq 50 \mathrm{MeV}$ and the inner core mass density $D_{0} \simeq 4 \times 10^{14} \mathrm{~g} / \mathrm{cm}^{3}$, the thermal number density of relativistic particles, say neutrinos or positrons, is negligible compared to the neutron number density. Therefore among the majoron interactions with $\psi=(e, p, n)$, the one with neutrons will play the most important role due to the huge neutron density inside the supernova core. Also majoron couplings to neutrinos readily indicate that the processes involving the heaviest neutrino flavor $\nu$ dominate over those without $\nu$.

For the effects of the matter-induced terms in eq.(10), we ignore those of the flavor off-diagonal term $(X / 2) b_{\mu} \bar{\nu} \gamma^{\mu} \gamma_{5} \nu^{\prime}$. The flavor-diagonal term $b_{\mu} \bar{\nu} \gamma^{\mu} \gamma_{5} \nu$ induces a polarization-dependent modification of both the dispersion relation and the wavefunction of $\nu$. We will take into account this modification only for the processes that come purely as a result of matter effecs ${ }^{[7]}$, viz $\nu \leftrightarrow \nu \phi$. The validity of these simplifications will be discussed in Appendix B.

The actual computation of the majoron luminosity and mean free path involves the temperature and density profile of the supernova core. Throughout this section, we will adopt the following simple model of the supernova core ${ }^{[24]}$. In the inner core of radius $r_{0} \simeq 10^{6} \mathrm{~cm}$, the temperature $T$ and the mass density $D$ are considered as being 
roughly constant,

$$
T(r) \simeq T_{0}, \quad D(r) \simeq D_{0}
$$

where $T_{0} \simeq 50 \mathrm{MeV}$ and $D_{0} \simeq 4 \times 10^{14} \mathrm{~g} / \mathrm{cm}^{3}$. Outside the inner core, i.e. $r \geq r_{0} \simeq$ $10^{6} \mathrm{~cm}$, we assume

$$
T(r) \simeq T_{0}\left(r_{0} / r\right)^{p} \quad, \quad D(r) \simeq D_{0}\left(r_{0} / r\right)^{3 p}
$$

where $p \simeq 2$.

Based on the simple model of the supernova core described above, one can obtain the surface temperature $T_{\nu}$ and the luminosity $L_{\nu}$ of the neutrino sphere in a straightforward way. At the surface of the neutrino sphere, the optical depth of the neutrinos is of order unity. Taking into account only the neutral current interaction with background nucleons that is the main source of neutrino trapping for $\nu_{\mu}$ or $\nu_{\tau}$, we find $T_{\nu} \simeq 6.2 \mathrm{MeV}$ and $L_{\nu} \simeq 10^{53} \mathrm{erg} / \mathrm{sec}$ for each neutrino flavor. These values are close to what one has obtained using more detailed models for supernova neutrino emission ${ }^{[16]}$, and thus imply that our simple model provides a reasonable description of the core region of SN1987A.

Throughout this paper, we will consider models in which the $B-L$ breaking scale $v$ is higher than the inner core temperature $T_{0}$,

$$
v \geq T_{0}
$$

in order to avoid any difficulty associated with the symmetry restoration of $U(1)_{B-L}$. Then the upper bound on the majoron-electron coupling $g_{\phi e e}=2 G_{F} A v m_{e}$ of eq.(2) leads to

$$
A \leq 1.7 \times 10^{-5}\left(\frac{G e V}{v}\right) .
$$

Note that the above bound is easily satisfied in generic singlet majoron models and does not give any severe constraint. 
In addition to the restriction of eq.(18), throughout the main body of this paper, we consider only the case that

$$
m_{\rho} \geq 6 T_{0}
$$

This additional restriction on $m_{\rho}$ will significantly simplify the computation of the majoron mean free path or luminosity. Note that since we are mainly interested in models with $v>T_{0}$, it does not significantly spoil the generality of our analysis. The case of $m_{\rho} \ll T_{0}$ will be briefly discussed in Appendix C.

\subsection{Majoron Mean Free Path}

For majoron interactions described by the effective lagrangian considered in Sec. 2, the following processes of majoron scattering or absorption ( see Fig. 3 for the Feynman diagrams for each process) give the potentially dominant contributions to the inverse mean free path of majorons:

$$
\begin{aligned}
& \text { (1.a) } \phi \nu \rightarrow \phi \nu, \quad(1 . b) \phi \phi \rightarrow \phi \phi, \quad(1 . c) \phi n \rightarrow \phi n \\
& \text { (1.d) } \phi \nu^{\prime} \rightarrow \nu, \quad(1 . e) \phi \nu \rightarrow \nu, \quad \text { (1.f) } \phi \phi \rightarrow \nu \nu .
\end{aligned}
$$

The process (1.e) is allowed as a result of background matter effects ${ }^{[7]}$ (for details, see Appendix B). There can be a resonant real $\rho$ production for the processes (1.b) and (1.f) in the case that the total four momentum of initial two majorons is on $\rho$-mass shell. Note that for majorons with a thermal distribution, unless $m_{\rho} \gg T_{0}$, there exists a significant probability of satisfying this on-shell condition.

The effects of other processes are negligible or at best are of the same order of magnitude as the processes considered above. In particular, as we will show explicitly, the processes that are associated with the majoron coupling to the axial vector (or 
vector) current of matter fermions, e.g. majoron capture via the inverse majoronneutron bremsstrahlung process $\phi n n \rightarrow n n$, or the process $\phi n \rightarrow \phi \phi n$ (see Fig. 3), do not affect majoron propagation at all because of the upper bound of eq.(19) on the coupling constant $A$.

The inverse mean free path of a majoron can be expressed as the sum of the contributions from each of the processes $(1 . a)-(1 . f)$,

$$
l_{\phi}^{-1}=\sum_{k=a}^{f} l_{\phi}^{-1}(1 . k),
$$

where $l_{\phi}^{-1}$ is given by

$$
l_{\phi}^{-1}(1 . k)=\frac{1}{2 E} \int d \Pi_{t} d \Pi_{f} f_{t}\left(p_{t}\right)(2 \pi)^{4} \delta^{4}\left(P_{i}-P_{f}\right)\left|M_{k}\right|^{2} \quad(k=a, b, \cdots, f) .
$$

Here $E$ corresponds to the energy of the incoming majoron, $d \Pi_{t}=d^{3} p_{t} /(2 \pi)^{3} 2 E_{t}$ is the phase space density of the target particle $t=\nu, \nu^{\prime}, n, \phi$ with energy-momentum $\left(E_{t}, p_{t}\right)$ and with the phase space distribution function $f_{t}$. Similarly $d \Pi_{f}=\Pi_{f} d^{3} p_{f} /(2 \pi)^{3} 2 E_{f}$ with the product over particles in the final state with energy-momentum $\left(E_{f}, p_{f}\right) . P_{i}$ and $P_{f}$ denote the total four momentum of the initial and final state respectively, and finally $\left|M_{k}\right|^{2}$ is the amplitude squared for the process $(1 . k)$ summed over initial and final spins (except for the matter-induced process (1.e) in which the initial and final neutrinos have opposite polarization) and includes the appropriate symmetry factor for identical particles in the final state. Inside the neutrino sphere, the neutrino phase space distribution function $f_{\nu}$ takes the form of the Fermi-Dirac distribution. For $f_{\phi}$, we will use the conventional Bose distribution regardless of whether majorons are in thermal equilibrium or not (e.g. freely escaping). This would result in a conservative estimate (overestimate) of the mean free path for freely escaping majorons. Due to the nonrelativistic nature of nucleons inside the supernova, the majoron mean free path is insensitive to the detailed form of $f_{n}$. 
Then by a straightforward calculation with $m_{\rho} \geq 6 T_{0}$, for incoming majorons with energy $E$, we find the majoron inverse mean free path (for each process) which is valid inside the neutrino sphere as

$$
\begin{aligned}
l_{\phi}^{-1}(\phi \nu \rightarrow \phi \nu) & \simeq 12\left(\frac{m}{M e V}\right)^{2}\left(\frac{G e V}{v}\right)^{4}\left(\frac{T}{T_{0}}\right)^{3} \mathrm{~cm}^{-1} \\
l_{\phi}^{-1}(\phi \phi \rightarrow \phi \phi) & \simeq 1.2 \times 10^{4}\left(\frac{G e V}{v}\right)^{4}\left(\frac{G e V}{m_{\rho}}\right)^{4}\left(\frac{E}{E_{0}}\right)^{3}\left(\frac{T}{T_{0}}\right)^{6} \mathrm{~cm}^{-1} \\
& +2.3 \times 10^{12}\left(\frac{m_{\rho}}{v}\right)^{2}\left(\frac{m_{\rho}}{G e V}\right)^{2}\left(\frac{E_{0}}{E}\right)^{2}\left(\frac{T}{T_{0}}\right) \exp \left(-\frac{m_{\rho}^{2}}{4 E T}\right) \mathrm{cm}^{-1} \\
l_{\phi}^{-1}(\phi n \rightarrow \phi n) & \simeq 2.4 \times 10^{-3} S^{2}\left(\frac{G e V}{m_{\rho}}\right)^{4}\left(\frac{E}{E_{0}}\right)^{4}\left(\frac{D}{D_{0}}\right) \mathrm{cm}^{-1} \\
l_{\phi}^{-1}\left(\phi \nu^{\prime} \rightarrow \nu\right) & \simeq 7.8 \times 10^{-1} X^{2}\left(\frac{m}{M e V}\right)^{4}\left(\frac{G e V}{v}\right)^{2}\left(\frac{E_{0}}{E}\right)^{2}\left(\frac{T}{T_{0}}\right) \mathrm{cm}^{-1} \\
l_{\phi}^{-1}(\phi \nu \rightarrow \nu) & \simeq 2 \times 10^{4}\left(\frac{m}{v}\right)^{2}\left(\frac{E_{0}}{E}\right)\left(\frac{T}{T_{0}}\right)\left(\frac{D}{D_{0}}\right) \ln \left(1+e^{-Z}\right) \mathrm{cm}^{-1} \\
l_{\phi}^{-1}(\phi \phi \rightarrow \nu \nu) & \simeq 15\left(\frac{m}{M e V}\right)^{2}\left(\frac{G e V}{v}\right)^{4}\left(\frac{T}{T_{0}}\right)^{3} \mathrm{~cm}^{-1}
\end{aligned}
$$

where $T$ and $D$ denote the temperature and mass density in the region of supernova under consideration, $T_{0} \simeq 50 \mathrm{MeV}$ is the inner core temperature while $D_{0} \simeq 4 \times$ $10^{14} \mathrm{~g} / \mathrm{cm}^{3}$ is the inner core mass density, $E_{0} \simeq 3 T_{0}$ corresponds to the average energy of relativistic particles inside the inner core, and $Z$ is given by

$$
Z=\frac{E}{2 T}\left(\left[1+4.4 \times 10^{2}(m / M e V)^{2}\left(E_{0} / E\right)\left(D_{0} / D\right)\right]^{1 / 2}-1\right) .
$$

Here all parameters correspond to those that appear in eqs.(10) and (14).

For the evaluation of the inverse mean free path associated with the scattering processes, we have used the following approximations for the functions defining crosssections (for notation and details, see Appendix A):

$$
F_{a} \simeq 0.5, F_{b} \simeq \frac{\pi}{\gamma} \delta(x-1)+\frac{14}{5} x^{3}, F_{c} \simeq \frac{4}{3} z^{2}, G \simeq 1+\frac{\pi}{\gamma} \delta(x-1),
$$

where $x=s / m_{\rho}^{2}, z=s\left(1-m_{n}^{2} / s\right)^{2} / 2 m_{\rho}^{2}$ and $\gamma$ is the total decay width of $\rho$ divided by $m_{\rho}$. For $m_{\nu} \ll T_{0} \ll m_{\rho}$, being the case of our interest, the $\rho$-decay into majorons gives 
a dominant contribution to the total width since the width for $\rho \rightarrow \nu \nu$ is suppressed by a factor $2 m_{\nu}^{2} / m_{\rho}^{2}$ compared to the one for $\rho \rightarrow \phi \phi$. Then we have $\gamma \simeq m_{\rho}^{2} / 32 \pi v^{2}$.

The delta-function in $F_{b}(G)$ denotes the production of a real $\rho$ which decays into two majorons (two neutrinos) in the process (1.b) ((1.f)). Since the dominant decay mode is the one into two majorons, the delta-function in $G$ is ignored in the computation of the inverse mean free path while the one in $F_{b}$ gives the Boltzmann-suppressed part in $l_{\phi}^{-1}(\phi \phi \rightarrow \phi \phi)$. Note that $x=s / m_{\rho}^{2}$ and thus the delta-function is the $\rho$-mass shell condition that is required for resonant real $\rho$-production.

Throughout the above computation of the inverse mean free path, we have ignored any background matter effects on neutrino propagation except for the process (1.e). This process is induced purely by matter effects and also gives the most important contribution to the inverse majoron mean free path among matter induced processes (see Appendix B).

For the processes involving neutrinos, it is assumed that neutrinos are in the relativistic regime. For nonrelativistic neutrinos, say neutrinos around the surface of the neutrino sphere with $T_{\nu} \ll m \leq 35 \mathrm{MeV}$, there will be an exponential suppression from the Boltzmann factor. Thus for nonrelativistic background neutrinos, naive use of the expressions in eq.(23) corresponds to an overestimate of the majoron inverse mean free path. Also in evaluating $l_{\phi}^{-1}(\phi \nu \rightarrow \nu)$, we have discarded a term that gives a minor but negative contribution and thus the result in eq.(23) is again a slightly overestimated one.

Our main purpose in this paper is to find a range of parameters which gives rise to the majoron luminosity greater than $3 \times 10^{53} \mathrm{erg} / \mathrm{sec}$. Then in any case, overestimating the inverse mean free path results in a conservative range of parameters and thus does 
not affect the validity of our final result. In the next two subsections, we will consider the majoron luminosity in both the cases of volume emission and blackbody surface emission using the mean free path derived here.

Finally, for completeness, let us consider the role of the majoron coupling to the vector or axial vector current of $\psi$, the only coupling whose effects are not included in the mean free path of eq.(23). The relevant processes associated with this coupling (see Fig. 3) are (1.g) $\phi n n \rightarrow n n$ and (1.h) $\phi n \rightarrow \phi \phi n$. Then

$$
\begin{aligned}
& l_{\phi}^{-1}(\phi n n \rightarrow n n) \simeq 3.4 \times 10\left(\frac{D}{D_{0}}\right)^{2}\left(\frac{A v}{G e V}\right)^{2}\left(\frac{E_{0}}{E}\right)^{0.5} \mathrm{~cm}^{-1} \\
& l_{\phi}^{-1}(\phi n \rightarrow \phi \phi n) \simeq 8.4 \times 10^{-10} A^{2}\left(\frac{D}{D_{0}}\right)\left(\frac{G e V}{v}\right)^{2}\left(\frac{G e V}{m_{\rho}}\right)^{4}\left(\frac{E}{E_{0}}\right)^{8} \mathrm{~cm}^{-1}
\end{aligned}
$$

These results, together with the astrophysical bound on $A$ of eq.(19), clearly indicate that the processes (1.g) and (1.h) give a negligible contribution to the inverse mean free path.

\subsection{Volume Emission}

With the majoron mean free path computed in the previous subsection, one can easily find the region of parameter space for which majorons freely escape from the supernova core. As an illustration, let us consider the case that $m \geq 10 \mathrm{KeV}$. Then majorons would freely stream out from the supernova core, i.e. $l_{\phi} \geq r_{0}$ inside the inner core, if all of the following conditions are satisfied:

$$
\begin{aligned}
& \left(\frac{m}{M e V}\right)\left(\frac{G e V}{v}\right)^{2} \leq 3.2 \times 10^{-4}, S\left(\frac{G e V}{m_{\rho}}\right)^{2} \leq 2 \times 10^{-2} \\
& \left(\frac{G e V}{v}\right)\left(\frac{G e V}{m_{\rho}}\right) \leq 3 \times 10^{-3}, X\left(\frac{m}{M e V}\right)^{2}\left(\frac{G e V}{v}\right) \leq 1.1 \times 10^{-3} \\
& \left(\frac{m}{M e V}\right)\left(\frac{G e V}{v}\right) \exp \left(-\frac{15 m}{M e V}\right) \leq 7.1 \times 10^{-3}
\end{aligned}
$$


Here we will compute the majoron luminosity for freely escaping majorons without further discussion of the corresponding conditions.

For freely escaping majorons, the total majoron luminosity is a simple sum of subluminosities that are due to the various production processes. Here we consider majoron production inside the inner core by the following three processes (see Fig. 4 for the corresponding Feynman diagrams) that are associated with the majoron couplings to neutrinos:

$$
(2 . a) \nu \nu \rightarrow \phi \phi, \quad(2 . b) \nu \rightarrow \nu^{\prime} \phi, \quad(2 . c) \nu \rightarrow \nu \phi
$$

Again the process (2.c) is induced as a result of background matter effects (see Appendix B) and gives the most important contribution to the majoron luminosity among matter induced processes. Note that, as long as majorons freely escape, one can safely isolate the effects of the above three processes from those of other processes.

The sub-luminosity from the inner core of radius $r_{0}$ is given by (for each of above processes)

$$
L_{\phi}(2 . j)=\int d V d \Pi_{i} d \Pi_{f} f_{i}\left(p_{i}\right)(2 \pi)^{4} \delta^{4}\left(P_{i}-P_{f}\right) E_{\phi}\left|M_{j}\right|^{2} \quad(j=a, b, c)
$$

where $d V$ denotes the infinitesimal volume inside the inner core, $d \Pi_{i}=\prod_{i} d^{3} p_{i} /(2 \pi)^{3} 2 E_{i}$ (with the product over particles in the initial state) and $d \Pi_{f}=\Pi_{f} d^{3} p_{f} /(2 \pi)^{3} 2 E_{f}$ (with the product over particles in the final state), $f_{i}$ is the phase space distribution function for the initial state, and $P_{i}$ and $P_{f}$ are the total four momentum of the initial and final state respectively. Here $E_{\phi}$ corresponds to the total energy of majorons in the final state and $\left|M_{j}\right|^{2}$ is the amplitude squared for the process (2.j) that includes the summation over initial and final spins and also symmetry facto $\mathrm{r}$ for identical particles. Again for the matter induced decay process $\nu \rightarrow \nu \phi$, initial and final neutrinos are oppositely polarized and thus no spin summation. 
Then using the effective lagrangian of eq.(10), a straightforward calculation leads to the following result for the luminosity from the inner core of radius $r_{0}$ :

$$
\begin{aligned}
L_{\phi}(\nu \nu \rightarrow \phi \phi) & \simeq 5.5 \times 10^{68}\left(\frac{m}{v}\right)^{2}\left(\frac{G e V}{v}\right)^{2}\left[1+\frac{2.4 \times 10^{-2}}{\gamma}\left(\frac{m_{\rho}}{T_{0}}\right)^{5.5} \exp \left(-\frac{m_{\rho}}{T_{0}}\right)\right] \\
L_{\phi}\left(\nu \rightarrow \nu^{\prime} \phi\right) & \simeq 7.5 \times 10^{61} X^{2}\left(\frac{m}{M e V}\right)^{4}\left(\frac{G e V}{v}\right)^{2} \\
L_{\phi}(\nu \rightarrow \nu \phi) & \simeq 4 \times 10^{53}\left(\frac{M e V}{m}\right)^{4}\left(\frac{G e V}{v}\right)^{2} \quad(\text { for } m \geq 95 \mathrm{Kev}) \\
& \simeq 4.8 \times 10^{65}\left(\frac{m}{v}\right)^{2} \quad(\text { for } m \leq 95 \mathrm{Kev}),
\end{aligned}
$$

where all the results are expressed in units of $\mathrm{erg} / \mathrm{sec}$.

In deriving the luminosity for the process $\nu \nu \rightarrow \phi \phi$, with the assumption $m_{\rho} \geq 6 T_{0}$, we have used an approximation for the function $G$ (for its definition, see Appendix A)

$$
G \simeq 1+\frac{\pi}{\gamma} \delta(x-1)
$$

The delta function in $G$ represents the production of real $\rho$ whose decay into two majorons eventually contributes to the energy loss via majoron emission. Note that it gives the Boltzmann-suppressed part in $L_{\phi}(\nu \nu \rightarrow \phi \phi)$. As in the computation of the majoron mean free path, we take into account the background matter effects only for the process $\nu \rightarrow \nu \phi$.

The above sub-luminosities of freely escaping majorons imply that the parameter range satisfying both the conditions of free escape and anyone of the following conditions gives a total luminosity greater than $3 \times 10^{53} \mathrm{erg} / \mathrm{sec:}$

$$
\begin{aligned}
&(30 . A) \nu \nu \rightarrow \phi \phi:\left(\frac{m}{v}\right)\left(\frac{G e V}{v}\right) \geq 2.3 \times 10^{-8} \\
&(30 . B) \nu \rightarrow \nu^{\prime} \phi:\left(\frac{\tau_{\nu}}{s e c}\right)\left(\frac{M e V}{m}\right) \leq 3.3 \times 10^{-5} \\
&(30 . C) \quad \nu \rightarrow \nu \phi:\left(\frac{m}{v}\right) \geq 7.9 \times 10^{-7} \quad(\text { for } m \leq 95 \mathrm{Kev}) \\
&\left(\frac{M e V}{m}\right)^{2}\left(\frac{G e V}{v}\right) \geq 8.7 \times 10^{-1} \quad(\text { for } m \geq 95 \mathrm{Kev})
\end{aligned}
$$


where the $\nu$-lifetime $\tau_{\nu}$ (for the decay $\nu \rightarrow \nu^{\prime} \phi$ as the main decay mode of $\nu$ ) in the vacuum (in the rest frame of $\nu$ ) is given by $\tau_{\nu}^{-1}=X^{2} m^{3} / 64 \pi v^{2}$. In the next subsection, we will consider the majoron luminosity when majorons are trapped and thermalized.

\subsection{Blackbody Surface Emission}

If $l_{\phi} \ll r_{0}$ inside the inner core, then majorons are trapped and form a thermal sphere. Here, using the mean free path derived in subsection 3.1 , we will determine the necessary conditions for the luminosity from the thermal majoron sphere to be larger than $3 \times 10^{53} \mathrm{erg} / \mathrm{sec}$.

The majoron luminosity in this case can be approximated by the simple formula of blackbody surface emission

$$
L_{\phi}=\frac{\pi^{3}}{30} R_{\phi}^{2} T_{\phi}^{4},
$$

where $R_{\phi}$ denotes the radius of the majoron sphere for which the majoron optical depth is of order unity, viz

$$
\int_{R_{\phi}}^{\infty} l_{\phi}^{-1} d r \simeq 1
$$

and $T_{\phi}$ is the corresponding surface temperature.

The majoron mean free path that appears in the optical depth of eq.(32) is that for a majoron emitted from a sphere of radius $R_{\phi}$, therefore with an average energy $E \simeq$ $3 T_{\phi}$, interacting with target particles outside $R_{\phi}$. Then in the computation we should take into account the fact that the number density of target particles, particularly neutrinos or majorons, does not follow a thermal distribution outside the region of thermal equilibrium. However a straightforward use of thermal distributions (with the temperature profile given in eq.(17)) over an arbitrarily large value of $r$ corresponds to overestimating the inverse mean free path and thus leads to a more conservative result 
for the parameter region forbidden by the requirement $L_{\phi} \leq 3 \times 10^{53} \mathrm{erg} / \mathrm{sec}$.

The blackbody surface luminosity of eq.(31), together with the temperature profile of eq.(17), implies that if

$$
T_{\phi} \geq 9.7 \mathrm{MeV}
$$

then $L_{\phi} \geq 3 \times 10^{53} \mathrm{erg} / \mathrm{sec}$. The parameter region for which majorons with average energy $3 T_{\phi} \geq 29 \mathrm{MeV}$ have optical depth of order unity can easily be derived from the mean free path in eq.(23). Then among the processes that contribute to the majoron inverse mean free path, majoron capture by the inverse decay process $\phi \nu^{\prime} \rightarrow \nu$ is special in the sense that the corresponding optical depth is an increasing function of $R_{\phi}$ inside the thermal sphere of $\nu^{\prime}$, while those for the other processes are decreasing functions.

Let us first consider the case in which majorons are trapped only by the process $\phi \nu^{\prime} \rightarrow \nu$. This would be possible if the contributions from the other processes to the inverse mean free path are negligible. Then it is easy to see that, for $\nu$ with $\tau_{\nu} \leq 10^{-7}(\mathrm{~m} / \mathrm{MeV}) \mathrm{sec}$, majorons are so strongly trapped that the resulting luminosity does not exceed $3 \times 10^{53} \mathrm{erg} / \mathrm{sec}$. For the general case in which the other processes make significant contributions to the majoron inverse mean free path, the majoron sphere has a surface temperature larger than $9.7 \mathrm{MeV}$ if all of the following conditions are satisfied:

$$
\begin{aligned}
& \text { (34.A) }(\phi \nu \rightarrow \phi \nu)+(\phi \phi \rightarrow \nu \nu):\left(\frac{m}{M e V}\right)\left(\frac{G e V}{v}\right)^{2} \leq 3.3 \times 10^{-3} \\
& (34 . B) \phi n \rightarrow \phi n: S\left(\frac{G e V}{m_{\rho}}\right)^{2} \leq 9.4 \\
& (34 . C) \phi \phi \rightarrow \phi \phi:\left(\frac{G e V}{v}\right)\left(\frac{G e V}{m_{\rho}}\right) \leq 1.8 \times 10^{-1} \\
& \text { (34.D) } \phi \nu^{\prime} \rightarrow \nu:\left(\frac{\tau_{\nu}}{s e c}\right)\left(\frac{M e V}{m}\right) \geq 10^{-6} \\
& \text { (34.E) } \phi \nu \rightarrow \nu:\left(\frac{m}{v}\right) \leq 1.7 \times 10^{-4} \quad(\text { for } m \leq 1 \mathrm{KeV}),
\end{aligned}
$$




$$
\left.\left(\frac{m}{v}\right)\left(\frac{K e V}{m}\right)^{1 / 2} \exp \left(-\frac{m}{2.4 K e V}\right) \leq 10^{-4} \text { (for } m \geq 1 \mathrm{KeV}\right)
$$

\section{Summary and Conclusion}

In the previous section, the range of parameters that is excluded by giving the majoron luminosity larger than $3 \times 10^{53} \mathrm{erg} / \mathrm{sec}$ has been found in the context of the effective lagrangian in Sec. 2. The results can be simply summarized as follows: the region of the parameter space where anyone of the conditions in eq.(30) and all of the conditions in eq.(34) are satisfied should be ruled out in view of the $\nu_{e}$-pulse from SN1987A since there $L_{\phi} \geq 3 \times 10^{53} \mathrm{erg} / \mathrm{sec}$.

We are interested in constraints on the mass $m$, the lifetime $\tau_{\nu}$ of $\nu_{\tau}$ (i.e $\nu$ in our notation) and the VEV $v$. Our analysis leads to the following results for $m$ and $\tau_{\nu}$ that are valid for generic majoron models with $v \geq T_{0} \simeq 50 \mathrm{Mev}$ and $m_{\rho} \geq 6 T_{0}$.

First of all, if $\tau_{\nu} \leq 10^{-7}(\mathrm{~m} / \mathrm{Mev}) \mathrm{sec}$, then $L_{\phi}$ does not exceed the critical value $3 \times 10^{53} \mathrm{erg} / \mathrm{sec}$ regardless of the values of $m$ and other parameters since majorons are strongly trapped by the process $\phi \nu^{\prime} \rightarrow \nu$. Therefore for this relatively short lived $\nu_{\tau}$, consideration of the energy release from SN1987A in the form of majorons does not give any constraint on the neutrino mass.

For the relatively long lived $\nu_{\tau}$, in particular when

$$
\tau_{\nu} \geq 10^{-6}\left(\frac{m}{M e V}\right) \mathrm{sec}
$$

a relatively wide range of $m$ depending on $v$ can be ruled out. If the parameters $\lambda_{3}, S$ 
and $v$ satisfy

$$
v \geq \operatorname{Max}\left\{0.23\left(\frac{S}{\lambda_{3}}\right)^{1 / 2}, \frac{2}{\lambda_{3}^{1 / 4}}\right\} \mathrm{GeV},
$$

then considering majoron production by the process $\nu \nu \rightarrow \phi \phi$ together with majoron trapping by $\phi \nu \rightarrow \phi \nu$ and $\phi \phi \rightarrow \nu \nu$ leads to the following forbidden range of $(v, m)$ that gives $L_{\phi} \geq 3 \times 10^{53} \mathrm{erg} / \mathrm{sec}(\operatorname{see}(30 . A)$ and $(34 . A))$ :

$$
2.3 \times 10^{-5} \leq\left(\frac{m}{M e V}\right)\left(\frac{G e V}{v}\right)^{2} \leq 3.3 \times 10^{-3} .
$$

Note that the restriction on $v$ of eq. (36) corresponds to (34.B) and (34.C) with $m_{\rho}^{2}=$ $2 \lambda_{3} v^{2}$ and is required to avoid strong majoron trapping by $\phi n \rightarrow \phi n$ or $\phi \phi \rightarrow \phi \phi$. In addition to the one of eq.(37), the matter-induced decay process $\nu \rightarrow \nu \phi$ provides the following forbidden range of $(v, m)$ (see $(30 . C))$ :

$$
7.9 \times 10^{-4}\left(\frac{v}{G e V}\right) \leq\left(\frac{m}{M e V}\right) \leq \operatorname{Min}\left\{\left(\frac{G e V}{v}\right)^{1 / 2}, 2.3 \times 10^{-5}\left(\frac{v}{G e V}\right)^{2}\right\}
$$

The region of the $v-m$ plane of eq.(37) and eq.(38) will be ruled out for $\tau_{\nu} \geq$ $10^{-6}(\mathrm{~m} / \mathrm{MeV}) \mathrm{sec}$, if $\lambda_{3}, S$ and $v$ satisfy eq.(36). In fact, eq.(36) is not so restrictive as long as $v \geq \mathrm{O}(1) \mathrm{GeV}$.

The forbidden region of the $v$ - $m$ plane given by eqs.(36), (37) and (38) is depicted in Fig. 5 for two different values of $\lambda_{3}, 1$ and $10^{-2}$, with the natural value $S \simeq 1$. For convenience, we also provide numerical results for the forbidden range of $m$ for several values of $v$ in Table 1 . These results are valid for generic majoron models in which $\nu_{\tau}$ is long-lived enough to satisfy $\tau_{\nu} \geq 10^{-6}(\mathrm{~m} / \mathrm{MeV})$ sec.

Finally we note that, for the cosmologically stable $\nu_{\tau}$, the region of the $v-m$ plane that is ruled out by the observation of the $\nu_{e}$-pulse from SN1987A can be excluded also from the consideration of the cosmological neutrino mass density. However it should be stressed that our result is valid also for the cosmologically unstable heavy 
$\nu_{\tau}$ that escapes all the existing phenomenological constraints including those from the cosmological and astrophysical considerations. In this regard, the analysis of the energy release from the supernova SN1987A by majoron emission provides a new constraint on the property of $\nu_{\tau}$ in generic singlet majoron models.

We thank R. Holman and L. Wolfenstein for useful dicussions. One of us (A. S.) thanks the Department of Physics of the Carnegie Mellon University, where most of this work has been done, for the hospitality extended to him. This work has been supported in part by the U.S. Department of Energy contract number DE-AC02-76ER03066. 


\section{Appendix A. Cross Sections}

In this appendix, we provide cross sections for the scattering processes that are relevant to our discussion of majoron emission from supernovae. A straightforward calculation leads us to the following expressions:

$$
\begin{aligned}
\sigma(\phi \nu \rightarrow \phi \nu) & =\frac{1}{16 \pi}\left(\frac{m^{2}}{v^{4}}\right) F_{a}(x, y), \\
\sigma(\phi \phi \rightarrow \phi \phi) & =\frac{1}{32 \pi}\left(\frac{m_{\rho}^{2}}{v^{4}}\right) F_{b}(x), \\
\sigma(\phi n \rightarrow \phi n) & =\frac{1}{4 \pi} S^{2} G_{F}^{2} m_{n}^{2} F_{c}(z), \\
\sigma(\nu \nu \rightarrow \phi \phi) & =\frac{1}{64 \pi}\left(\frac{m^{2}}{v^{4}}\right) G(x, y) \\
\sigma(\phi \phi \rightarrow \nu \nu) & =\frac{1}{16 \pi}\left(\frac{m^{2}}{v^{4}}\right)(1-4 y) G(x, y)
\end{aligned}
$$

where $x=s / m_{\rho}^{2}, y=m^{2} / s$ and $z=s\left(1-m_{n}^{2} / s\right)^{2} / 2 m_{\rho}^{2}$.

The functions in the above cross sections are given by

$$
\begin{aligned}
F_{a}= & \frac{\ln \left[1+x(1-y)^{2}\right]}{x^{2}(1-y)^{2}}-\frac{1}{x\left[1+x(1-y)^{2}\right]}+\frac{5 y}{2}+\frac{y^{2}}{2}+\frac{2 y}{x(1-y)}+ \\
& \frac{4 y}{1+x(1-y)^{2}}-\frac{y[x(1-y)-1] \ln y}{(1-y)[x(1-y)+1]}-\frac{2 y[1+2 x(1-y)]^{2} \ln \left[1+x(1-y)^{2}\right]}{x^{2}(1-y)^{3}[1+x(1-y)]} \\
F_{b}= & \frac{6 x^{2}-4 x-x^{3}+4(1-x) \ln (1+x)}{(1-x)^{2}+\gamma^{2}}+x-4+\frac{2(2 x+3)}{x(x+1)}+\frac{4\left(x^{2}-3\right) \ln (1+x)}{x^{2}(x+2)} \\
F_{c}= & \frac{2(1+z)}{1+2 z}-\frac{1}{z} \ln (1+2 z) \\
G= & \frac{\sqrt{1-4 y}}{(1-x)^{2}+\gamma^{2}}\left[1+\frac{y(x-1)(x+1)}{1-4 y}\left\{\frac{2}{\sqrt{1-4 y}} \ln \left(\frac{1+\sqrt{1-4 y}}{1-\sqrt{1-4 y}}\right)-4\right\}\right] .
\end{aligned}
$$

where $\gamma=\Gamma / m_{\rho} \simeq m_{\rho}^{2} / 32 \pi v^{2}$. Here $\Gamma$ is the total decay width of the $\rho$ which, in our case of $m_{\nu} / m_{\rho} \ll 1$, is dominated by the decay into two majorons. Here the nonrelativistic limit is taken for target neutrons in the process $\phi n \rightarrow \phi n$. The dependence on $\gamma$ in $F_{b}$ and $G$ represents the effect of the production of a real $\rho$ which eventually decays into the corresponding final states, two majorons or two neutrinos. 
The majoron inverse mean free path and volume luminosity considered in Sec. 3 involve the thermal average of the above functions. There we have adopted the following approximations:

$$
F_{a} \simeq 0.5, \quad F_{b} \simeq \frac{\pi}{\gamma} \delta(x-1)+\frac{14}{5} x^{3}, \quad F_{c} \simeq \frac{4}{3} z^{2}, \quad G \simeq 1+\frac{\pi}{\gamma} \delta(x-1) .
$$

The delta functions in these approximations denote the production of a real $\rho$ and are embedded in the full expressions of $F_{i}(i=a, b, c)$ and $G$ through the relation $1 /\left(\omega^{2}+\epsilon^{2}\right) \simeq \pi \delta(\omega) / \epsilon(\epsilon \ll 1)$. The remaining terms correspond to the values in the limit of $x \ll 1$ and $z \ll 1$.

In order to justify the limit of $x \ll 1$ and $z \ll 1$, let us consider the average values of the variables $x, y$ and $z$ inside supernovae. For relativistic neutrinos, these averages can be estimated as:

$$
\begin{aligned}
& \langle x\rangle=\frac{\langle s\rangle}{m_{\rho}^{2}} \simeq\left(\frac{4.2 T}{m_{\rho}}\right)^{2} \\
& \langle y\rangle=\frac{m_{\nu}^{2}}{\langle s\rangle} \simeq\left(\frac{m}{4.2 T}\right)^{2} \\
& \langle z\rangle=\frac{\left\langle s\left(1-m_{n}^{2} / s\right)^{2}\right\rangle}{2 m_{\rho}^{2}} \simeq\left(\frac{4.2 T}{m_{\rho}}\right)^{2} .
\end{aligned}
$$

Then for $m_{\rho} \geq 6 T_{0}$, which has been assumed throughout the main body of this paper, we have $\langle x\rangle \leq 0.5$ and $\langle z\rangle \leq 0.5$ and therefore the expressions obtained in the limit of $x \ll 1$ and $z \ll 1$ can be considered as a reasonable approximation. Note that the delta functions of the real $\rho$ production which give a nonzero contribution only when $x=1$ are also included because of their potential importance due to the large phase space. 


\section{Appendix B. Matter Induced Neutrino Decay}

The effective lagrangian of eq.(10) expressed in terms of the mass eigenstates in the vacuum includes terms that represent the effects of the coherent neutrino scattering off nucleons inside supernovae,

$$
\frac{1}{2} b_{\mu}\left(\bar{\nu} \gamma^{\mu} \gamma_{5}+\bar{\nu}^{\prime} \gamma^{\mu} \gamma_{5} \nu^{\prime}-X \bar{\nu} \gamma^{\mu} \gamma_{5} \nu^{\prime}\right)
$$

Then neutrinos propagating inside supernovae correspond to the energy-eigenstates of the effective Hamiltonian including the above matter-induced terms.

Let us, for a moment, neglect the last term that is flavor off-diagonal in the basis of mass eigenstates in the vacuum, and discuss the effect of the flavor-diagonal term of $\nu$. Then in the rest frame of the background matter for which $b_{\mu}=b \delta_{\mu}^{0}\left(b=G_{F} Y_{n} / \sqrt{2}\right)$, the Dirac equation for $\nu$ can be written as

$$
\left(\vec{\alpha} \cdot \vec{p}+m \gamma_{0}+b \gamma_{5}\right) \nu=E(\vec{p}) \nu
$$

and gives rise to the following polarization-dependent dispersion relation,

$$
E_{ \pm}^{2}=(p \pm b)^{2}+m^{2}
$$

where \pm denotes polarization and $p=|\vec{p}|$. In the chiral representation of $\gamma$-matrices, the energy eigenstates $\nu_{ \pm}$with the eigenvalues $E_{ \pm}$are given by

$$
\nu_{+}^{T}=\left(\chi_{+},-m \chi_{+} /\left(E_{+}+p+b\right)\right), \nu_{-}^{T}=\left(-m \chi_{-} /\left(E_{-}+p-b\right), \chi_{-}\right),
$$

where $\chi_{ \pm}$is the two-component spinor satisfying

$$
\vec{p} \cdot \vec{\sigma} \chi_{ \pm}= \pm p \chi_{ \pm}, \quad 2 p \chi_{ \pm} \chi_{ \pm}^{\dagger}=\left(E_{ \pm}+p \pm b\right)(p \pm \vec{p} \cdot \vec{\sigma})
$$

As was noted in Ref. 7, the above polarization-dependent modification of the dispersion relation and the resulting energy splitting between the states of different 
polarizations make the processes $\nu_{+} \leftrightarrow \nu_{-} \phi$, not allowed in the vacuum, possible inside supernovae. The coupling that is responsible for the decay is the flavor diagonal one, $\partial_{\mu}(\phi / v) \bar{\nu} \gamma^{\mu} \gamma_{5} \nu$. The corresponding decay rate can be easily computed using the dispersion relation and the energy eigenstates derived above. Although important for those not allowed in the vacuum, these matter effects can be ignored for the processes allowed in the vacuum, e.g. $\nu \nu \rightarrow \phi \phi$ or $\phi \nu \rightarrow \phi \nu$, as long as $p \gg b$.

Let us consider the possible role of the matter-induced flavor off-diagonal term $(X / 2) b_{\mu} \bar{\nu} \gamma^{\mu} \gamma_{5} \nu^{\prime}$. Then the dispersion relation and the energy eigenstates that are derived without taking into account this term will be modified. For example, the energy eigenstates with this term correspond to linear combinations of the $\nu$-state and $\nu^{\prime}$-state with same polarization where the mixing angle for each polarization is given by

$$
\tan 2 \theta_{ \pm} \simeq \mp \frac{2 X b p}{m^{2}}
$$

However it can be easily checked that any modification induced by the matter-induced flavor off-diagonal term is not important at all for the majoron production and trapping inside supernovae and thus we can ignore it throughout the analysis.

One might think that we do not have to consider the original flavor off-diagonal majoron coupling $X\left(\partial_{\mu} \phi / 2 v\right) \bar{\nu} \gamma^{\mu} \gamma_{5} \nu^{\prime}$ and the associated processes $\nu \leftrightarrow \nu^{\prime} \phi$, since we already include the similar processes $\nu \leftrightarrow \nu \phi$ that are induced by matter effects. However for $m^{2} \gg b p$, it turns out that the process $\phi \nu^{\prime} \rightarrow \nu$ can dominate over the matter-induced $\phi \nu \rightarrow \nu$ and thus must be included seperately. 


\section{Appendix C. The Case of a Light $\rho$}

In Ref. 6 it was argued that for the range of the majoron Yukawa coupling $h=m / v$, $10^{-5} \leq h \leq 10^{-4}$, the energy loss of supernovae by majoron emission dominates over the one by neutrino emission. The processes considered there were $\nu \nu \rightarrow \phi \phi$ and $\phi \nu \rightarrow \phi \nu$. Our result of the excluded parameter region in Sec. 3 does not involve this range of $h$. Here we will argue that the result of Ref. 6 is valid only for an extremely light $\rho$ whose mass is much smaller than $T_{0}$, i.e. valid only for a parameter region far outside the one, viz $m_{\rho} \geq 6 T_{0}$, considered throughout the main body of this paper.

The authors of Ref. 6 used the majoron-neutrino coupling $\phi \bar{\nu} i \gamma_{5} \nu$ in computing the amplitudes for the processes $\nu \nu \rightarrow \phi \phi$ and $\phi \nu \rightarrow \phi \nu$. Then they made the assumption that the other majoron couplings are weak enough to be ignored. As a consequence all diagrams involving $\rho$, as a real or virtual particle, were neglected. Therefore in order to find the parameter region where the assumption made in Ref. 6 is valid, we shoul $d$ use the $\gamma_{5}$-coupling scheme of the majoron. Note that throughout the main body of this paper, we have used the derivative coupling scheme that is achieved by the exponential parameterization of $\sigma$ of eq.(6) and the majoron dependent phase rotation of neutrinos of eq.(8). (We will abreviate the whole procedure that gives the derivative coupling scheme of the majoron as EP.)

In fact one can arrive at the $\gamma_{5}$-coupling with the more conventional linear parameterization (LP) of $\sigma$ :

$$
\sigma=\frac{1}{\sqrt{2}}(v+\rho+i \phi) .
$$

As is well known, any on-shell amplitude at both the exact and tree level is independent of the parameterization of field variables, and thus the effective lagrangian of $\phi$ and $\rho$ in LP is equivalent to the one in EP even though different in its explicit form. However 
in order to see the equivalence, one must be careful to include all the relevant Feynman diagrams that contribute to the process.

Let us consider the $\phi-\rho$ and $\phi-\phi$ couplings in the $\gamma_{5}$-coupling scheme of LP. Then it is rather easy to see that they vanish in the limit $\lambda_{3} \rightarrow 0$. This implies that the assumption made in Ref. 6 can hold true if $\lambda_{3}$ (or equivalently $\left(m_{\rho} / v\right)^{2}=2 \lambda_{3}$ ) is small enough.

A more detailed analysis leads to the following results. It turns out that one of the conditions for the validity of the assumption made in Ref. 6 is

$$
m_{\rho}^{2} \leq 4 m T_{\nu}
$$

where $T_{\nu} \simeq 6.2 \mathrm{MeV}$ denotes the surface temperature of the $\nu_{\tau}$-sphere. Then for this light $\rho$, the majoron inverse mean free path and volume luminosity are given by:

$$
\begin{aligned}
l_{\phi}^{-1}(\phi \nu \rightarrow \phi \nu) & \simeq 7 \times 10^{8}\left(\frac{m}{v}\right)^{4}\left(\frac{T}{T_{0}}\right)^{2}\left(\frac{E_{0}}{E}\right) \ln \left(\frac{7 T E}{m^{2}}\right) \mathrm{cm}^{-1} \\
l_{\phi}^{-1}(\phi \phi \rightarrow \rho) & \simeq 5.6 \times 10^{9}\left(\frac{m_{\rho}}{v}\right)^{4}\left(\frac{v}{T_{0}}\right)^{2}\left(\frac{E_{0}}{E}\right)\left(\frac{3 T}{E}\right) \ln \left(\frac{4 E T}{m_{\rho}^{2}}\right) \mathrm{cm}^{-1} \\
l_{\phi}^{-1}(\phi n \rightarrow \phi n) & \simeq 9.2 \times 10^{-1} S^{2}\left(\frac{D}{D_{0}}\right) \mathrm{cm}^{-1} \\
L_{\phi}(\nu \nu \rightarrow \phi \phi) & \simeq 6.4 \times 10^{70}\left(\frac{m}{v}\right)^{4}\left[\ln \left(\frac{T_{0}}{m}\right)+\frac{7.5 \times 10^{-2}}{\gamma}\left(\frac{m_{\rho}}{m}\right)^{2}\left(\frac{m_{\rho}}{T_{0}}\right)^{2}\right]
\end{aligned}
$$

where all the notation is the same as that used in eqs.(23) and (28). For the processes $\nu \leftrightarrow \nu^{\prime} \phi$ and $\nu \leftrightarrow \nu \phi$, the results are independent of $m_{\rho}$ and thus are same as those in eq. (23).

In the case of $m_{\rho}^{2} \leq 4 m T_{\nu}$, we have $\langle x\rangle^{2} \geq\langle y\rangle^{-1} \gg 1$ (see Appendix A) and then one can make the following approximations for the functions defining the cross-sections:

$$
F_{a} \simeq y(1-\ln y), F_{b} \simeq \frac{\pi}{\gamma} \delta(x-1), F_{c} \simeq 1, G \simeq-2 y(\ln y+2)+\frac{\pi}{\gamma} \delta(x-1)
$$


For $v \geq T_{0}$, the real $\rho$ production gives a dominant contribution to $F_{b}$ due to its large phase space.

Now one can easily see that if we ignore the potential trapping due to the processes $\phi \phi \rightarrow \rho, \phi n \rightarrow \phi n, \phi \nu^{\prime} \rightarrow \nu$ and $\phi \nu \rightarrow \nu$, then the above mean free path $l_{\phi}(\phi \nu \rightarrow \phi \nu)$ and the volume luminosity $L_{\phi}(\nu \nu \rightarrow \phi \phi)$ lead to a conclusion about $h=m / v$ that is similar to the one of Ref. 6 . However, unless

$$
\frac{m_{\rho}}{v} \leq 10^{-4}, S \leq 10^{-3}
$$

majorons will be so strongly trapped due to $\phi-\phi$ and $\phi-n$ scattering that the luminosity is less than $3 \times 10^{53} \mathrm{erg} / \mathrm{sec}$ independently of the value of $h$. 


\section{References}

[1] K. Hirata, et al., Phys. Rev. Lett. 58, 1490 (1987); R. M. Bionta, et al., Phys. Rev. Lett. 58, 1494 (1987).

[2] J. Ellis and K. A. Olive, Phys. Lett. 193B, 525 (1987)

[3] J. M. Lattimer and J. Cooperstein, Phys. Rev. Lett. 61, 23 (1988); R. Barbieri and R. N. Mohapatra, Phys. Rev. Lett. 61, 27 (1988).

[4] E. W. Kolb and M. S. Turner, Phys. Rev. D36, 2895 (1987).

[5] Y. Aharonov, F. T. Avignone, and S. Nussinov, Phys. Lett. 220B, 122 (1988); Phys. Rev. D37, 1360 (1988); Phys. Rev. D39, 985 (1989).

[6] K. Choi, C. W. Kim, J. Kim, and W. P. Lam, Phys. Rev. D37, 3225 (1988); J. A. Grifols, E. Masso and S. Peris, Phys. Lett. 215B, 593 (1988).

[7] Z. G. Berezhiani and M. I. Vysotsky, Phys. Lett. 199B, 281 (1987); Z. G. Berezhiani and A. Yu. Smirnov, Phys. Lett. 220B, 279 (1989).

[8] A. Manohar, Phys. Lett. 192B, 217 (1987).

[9] Y. Chikashige, R. N. Mohapatra, and R. D. Peccei, Phys. Lett. 98B, 265 (1981).

[10] K. Choi and A. Santamaria, "Dark Matter Neutrinos and Natural Mass Hierarchy in Singlet-Triplet Majoron Model", Carnegie Mellon University Report CMUHEP89-22 and MPI Report MPI-PAE/PTh 74/89 (1989).

[11] D. Chang, W. Y. Keung, and P. B. Pal, Phys. Rev. Lett. 61, 2420 (1988); A. Santamaria, Phys. Rev. D39, 2715 (1989). Hyperchargeless triplet majorons considered also in these papers behave like singlet majorons in many respects. In 
particular they do not couple to electrons at tree level nor have the transition into $\rho$ by the neutral current interaction. As a result, the astrophysical bound on the majoron-electron coupling or the precise measurement of the decay width of $Z^{0}$ does not provide a useful constraint on the model. Furthermore such majorons will not be trapped by gauge interactions inside the supernova core.

[12] G. B. Gelmini and M. Roncadelli, Phys. Lett. 99B, 411 (1981) H. M. Georgi, S. L. Glashow, and S. Nussinov, Nucl. Phys. B193, 297 (1981).

[13] C. S. Aulakh and R. N. Mohapatra, Phys. Lett. 119B, 136 (1982); J. F. Nieves, Phys. Lett. 137B, 67 (1984); A. Santamaria and J. W. F. Valle, Phys. Lett. 195B, 423 (1987); S. Bertolini and A. Santamaria, Nucl. Phys. B310, 714 (1988); A. Santamaria and J. W. F. Valle, Phys. Rev. Lett. 60, 397 (1988); Phys. Rev. D39, 1780 (1989).

[14] D. A. Dicus, E. W. Kolb, V. L. Teplitz, and R. V. Wagoner, Phys. Rev. D22, 839 (1980); M. Fukugita, S. Watamura, and M. Yoshimura, Phys. Rev. D26, 1840 (1982).

[15] D. S. P. Dearborn, D. N. Schramm, and G. Steigman, Phys. Rev. Lett. 56, 26 (1986); G. G. Rafelt, Phys. Rev. D33, 897 (1986); A. Pantziris and K. Kang, Phys. Rev. D33, 3509 (1986); H. Y. Cheng, Phys. Rev. D36, 1649 (1987).

[16] R. Mayle, J. R. Wilson, and D. N. Schramm, Astrophys. J. 318, 288 (1987); A. Burrows and J. M. Lattimer, Astrophys. J. 307, 178 (1986).

[17] Axion emission from supernova associated with the axion-nucleon bremsstrahlung process has been considered by; R. Mayle, et al., Phys. Lett. 203B, 188 (1988); G. G. Raffelt and D. Seckel, Phys. Rev. Lett. 60, 1793 (1988); M. S. Turner, Phys. Rev. Lett. 60, 1797 (1988). 
[18] A. Burrows, M. S. Turner, and R. P. Brinkmann, Phys. Rev. D39, 1020 (1989) have incorporated axion emission into realistic numerical models of supernova cooling. The result from this careful analysis is in good agreement with that from the more naive approach in ref.[17].

[19] See, for a recent review, E. W. Kolb, D. N. Schramm, and M. S. Turner, FermilabPub-89/97-A (1989).

[20] S. L. Glashow, Phys. Lett. 187B, 367 (1987).

[21] E. D. Carlson and L. J. Hall, " $\nu_{\mu}$ and $\nu_{\tau}$ as Dark Matter", UCB-PTH-89/10 (1989).

[22] See also ref.[10].

[23] L. Wolfenstein, Phys. Rev. D17, 2369 (1978); P. Mikheyev and A. Yu. Smirnov, Sov. J. Nucl. Phys. 42, 913 (1985).

[24] A similar model has been used by M. S. Turner, Phys. Rev. Lett. 60, 1797 (1988). 


\section{Figure and Table Captions}

Table 1. Excluded range of the neutrino mass $m$ for a given value of the $B-L$ breaking scale $v$.

Figure 1. Feynman diagram for the effective coupling of $\phi$ to the bilinear current of $\psi$. The internal fermion loop denotes neutrinos and the four-fermion vertex is due to the standard weak interactions.

Figure 2. Feynman diagram for the effective coupling of $\rho$ to the bilinear scalar density of $\psi$. The double-dashed line denotes the physical Higgs in the weak-doublet $H_{i}$ whose mixing with $\rho$ is induced by the $\lambda_{2}$-quartic coupling in the Higgs potential.

Figure 3. Feynman diagrams for the processes contributing to the majoron inverse mean free path. The dashed line denotes $\phi$ while the double-line is for $\rho$.

Figure 4. Feynman diagrams for the processes producing majorons inside the inner core of supernovae.

Figure 5. The region (inside the curve) of the $v-m$ plane excluded by giving $L_{\phi} \geq$ $3 \times 10^{53} \mathrm{erg} / \mathrm{sec}$. The result is valid for generic singlet majoron models in which $\tau_{\nu} \geq$ $10^{-6}(\mathrm{~m} / \mathrm{MeV})$. The line $A I$ comes from the majoron trapping by $\phi \nu \rightarrow \phi \nu$ and $\phi \phi \rightarrow$ $\nu \nu, B C$ and $E H$ come from the volume emission via $\nu \nu \rightarrow \phi \phi$ while $C D E$ is for the matter-induced $\nu \rightarrow \nu \phi$, and finally $F G(H I)$ comes from the possibility of majoron trapping by $\phi \phi \rightarrow \phi \phi$ when $\lambda_{3} \simeq 10^{-2}\left(\lambda_{3} \simeq 1\right)$. 


\begin{tabular}{||c|c||}
\hline$v(\mathrm{GeV})$ & $m(\mathrm{KeV})$ \\
\hline 8 & $1.5-210$ \\
\hline 10 & $2.3-330$ \\
\hline 20 & $9.2-1300$ \\
\hline 30 & $21-3000$ \\
\hline 50 & $40-8300$ \\
\hline 80 & $63-120$ and $150-21000$ \\
\hline 100 & $79-110$ and $230-33000$ \\
\hline 200 & $920-35000$ \\
\hline 300 & $2100-35000$ \\
\hline 500 & $5800-35000$ \\
\hline
\end{tabular}

Table 1. 


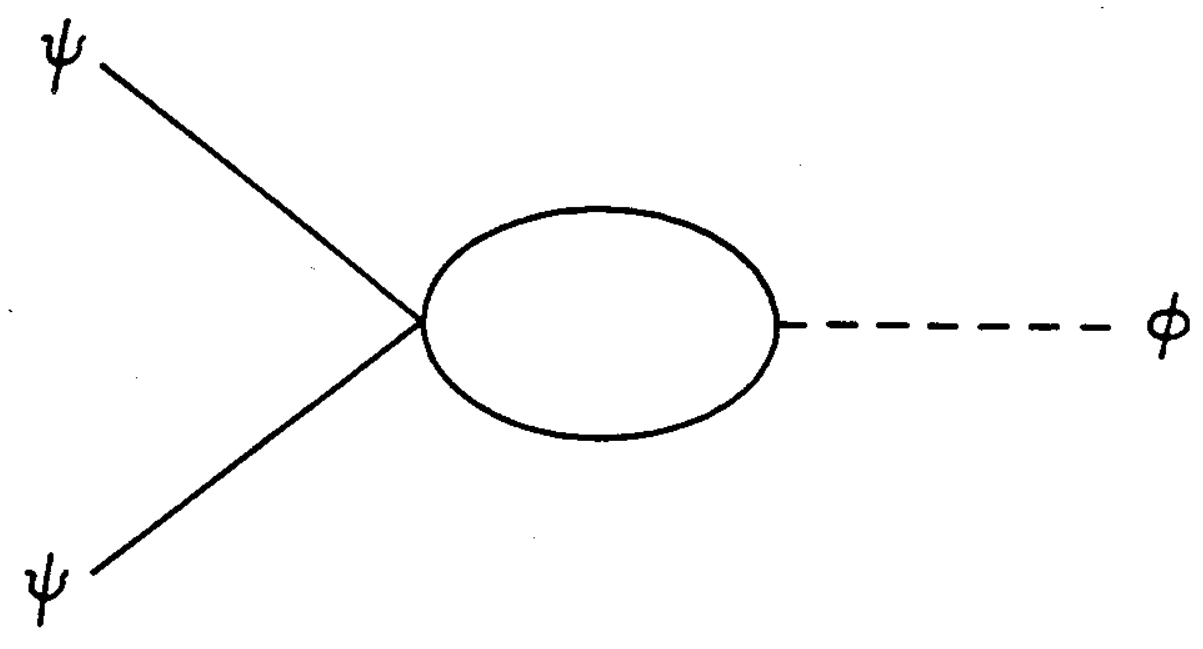

FIG.1

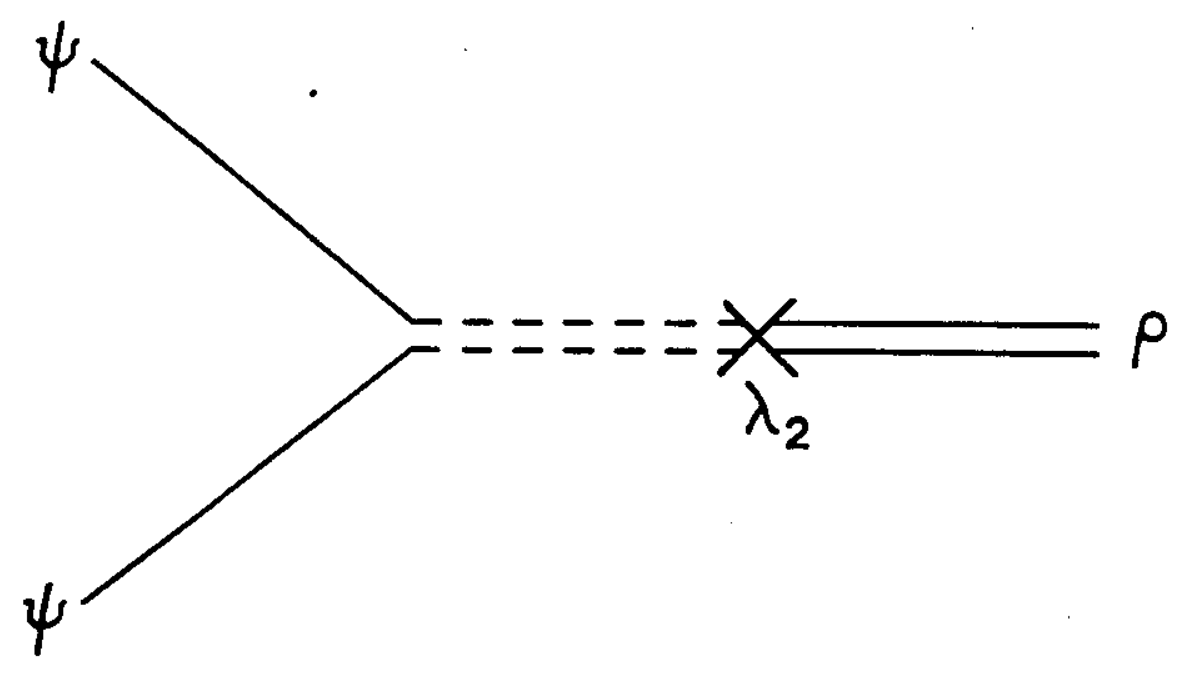

FIG. 2 

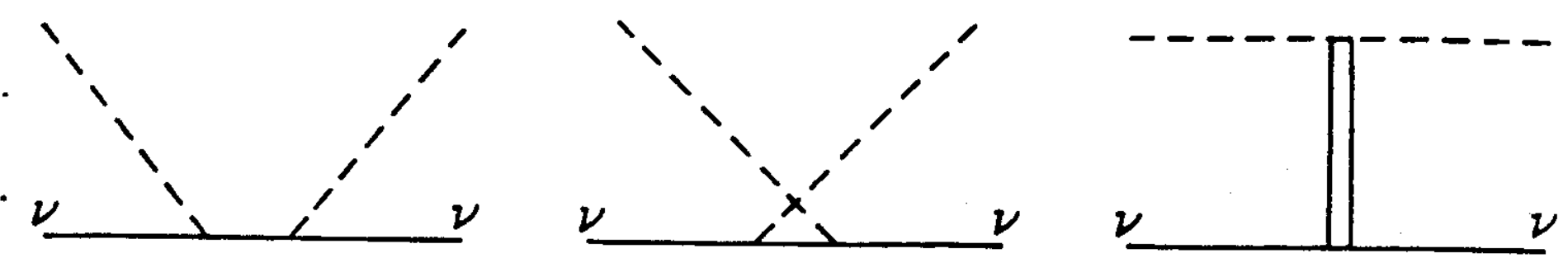

(a)
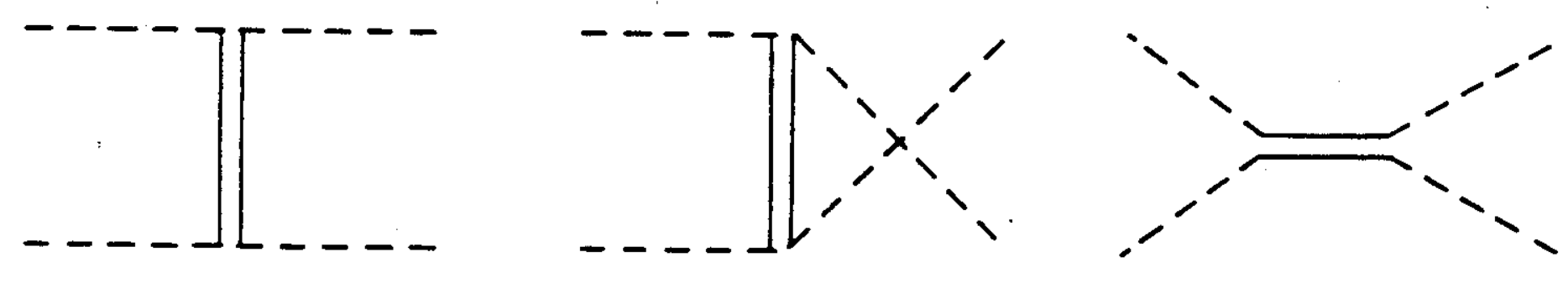

(b)

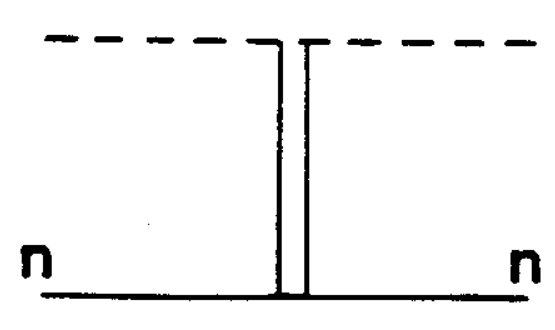

(C)

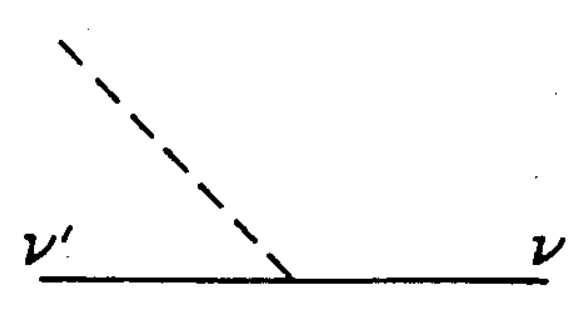

(d)

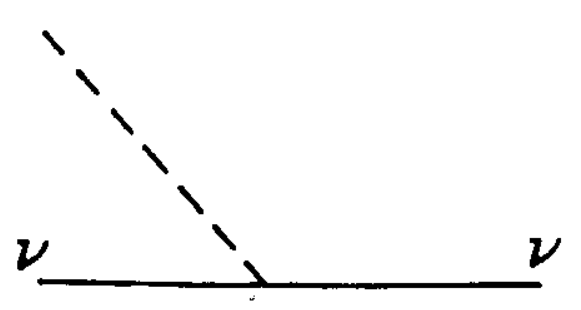

(e)

FIG. 3 

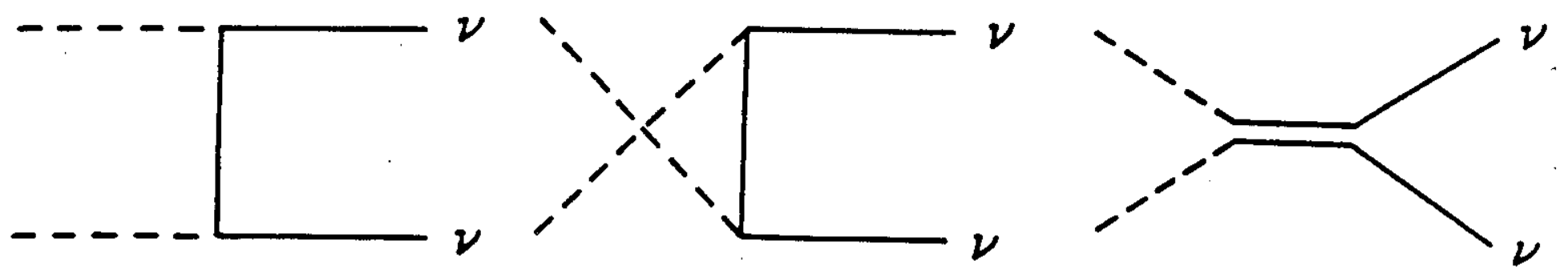

(f)

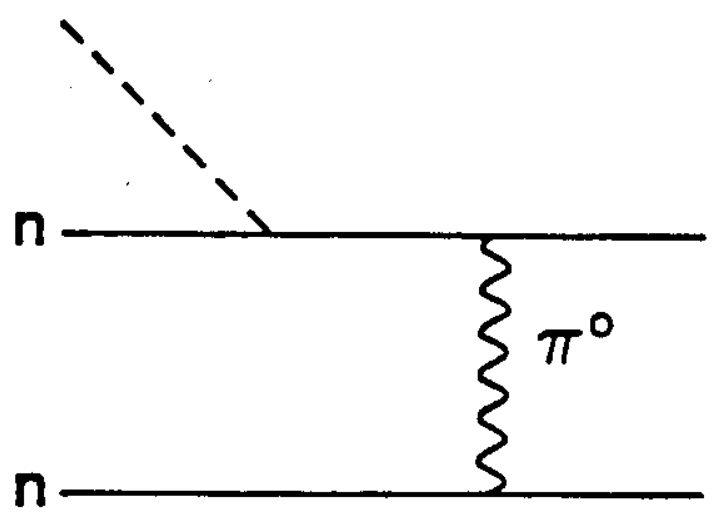

+ permutations

(g)
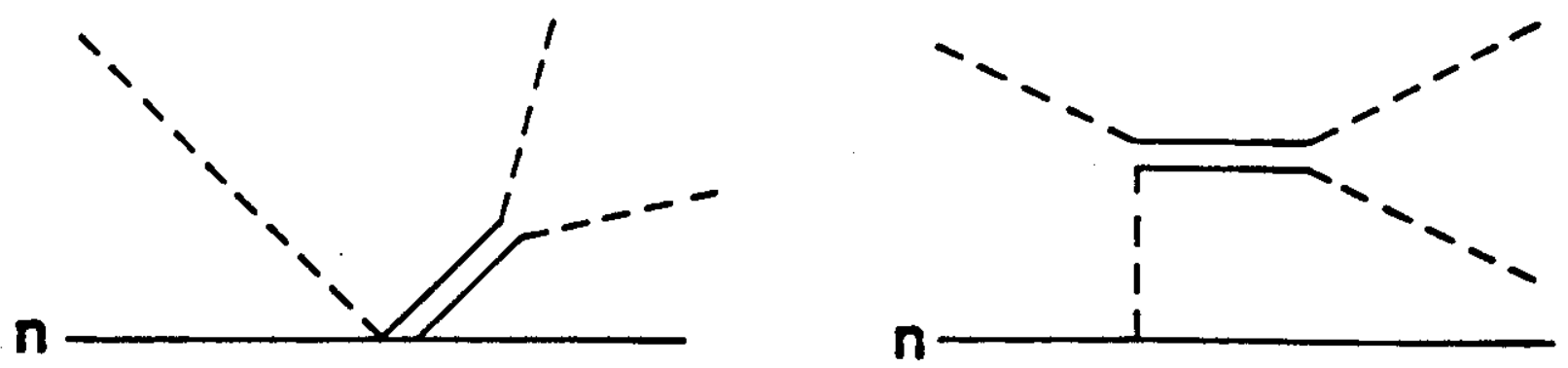

(h)

FIG. 3 

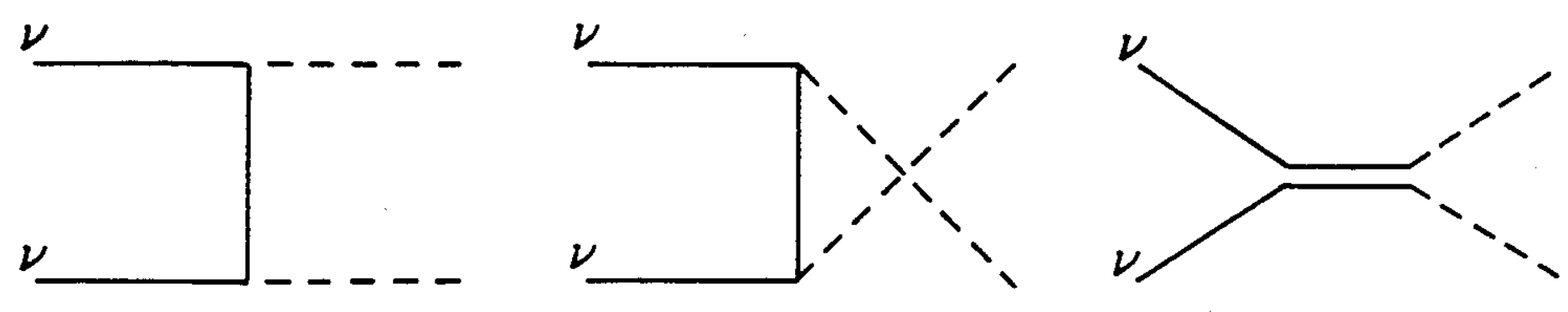

(a)

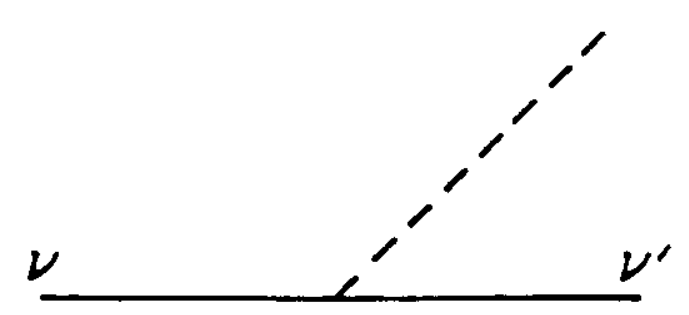

(b)

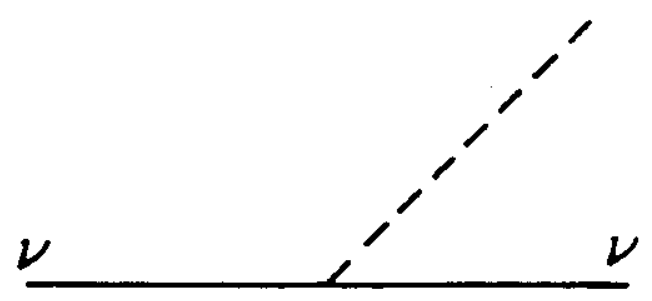

(C)

FIG. 4 
